\title{
A STUDY OF COLLECTIVELY COINCIDENCE POINTS AND MAXIMAL TYPE ELEMENTS
}

\author{
DONAL O'REGAN \\ School of Mathematical and Statistical Sciences, National University of Ireland, \\ Galway, Ireland.
}

\begin{abstract}
We present some new collectively fixed and coincidence results for families of maps which enables us to establish maximal element type results.
\end{abstract}

AMS (MOS) Subject Classification. 47H10, 54H25.

Key Words and Phrases. Continuous selections, fixed point theory, coincidence theory, maximal elements.

\section{INTRODUCTION}

Motivated by ideas in $[4,12,13]$ in this paper we present some new collectively fixed and coincidence type results. Then using these results we establish some new maximal type element theorems for families of majorized type maps $[4,6]$ in the compact setting. In this paper we discuss the $\Phi^{\star}$ maps from the literature [2] and also admissible maps in the sense of of Gorniewicz [9]. We present collectively coincidence results bewteen classes of maps (the first result is between the same classes and the second result is between different classes).

Now we describe the maps considered in this paper. Let $H$ be the Cech homology functor with compact carriers and coefficients in the field of rational numbers $K$ from the category of Hausdorff topological spaces and continuous maps to the category of graded vector spaces and linear maps of degree zero. Thus $H(X)=\left\{H_{q}(X)\right\}$ (here $X$ is a Hausdorff topological space) is a graded vector space, $H_{q}(X)$ being the $q^{-}$ dimensional $\breve{C e c h}$ homology group with compact carriers of $X$. For a continuous map $f: X \rightarrow X, H(f)$ is the induced linear map $f_{\star}=\left\{f_{\star q}\right\}$ where $f_{\star q}: H_{q}(X) \rightarrow$ $H_{q}(X)$. A space $X$ is acyclic if $X$ is nonempty, $H_{q}(X)=0$ for every $q \geq 1$, and $H_{0}(X) \approx K$.

Received September 20, 2021

$\$ 15.00$ (C) Dynamic Publishers, Inc. www.dynamicpublishers.org .
ISSN1056-2176(Print); ISSN 2693-5295 (online) https://doi.org/10.46719/dsa202130.12.01 
Let $X, Y$ and $\Gamma$ be Hausdorff topological spaces. A continuous single valued map $p: \Gamma \rightarrow X$ is called a Vietoris map (written $p: \Gamma \Rightarrow X$ ) if the following two conditions are satisfied:

(i). for each $x \in X$, the set $p^{-1}(x)$ is acyclic

(ii). $\quad p$ is a perfect map i.e. $p$ is closed and for every $x \in X$ the set $p^{-1}(x)$ is nonempty and compact.

Let $\phi: X \rightarrow Y$ be a multivalued map (note for each $x \in X$ we assume $\phi(x)$ is a nonempty subset of $Y$ ). A pair $(p, q)$ of single valued continuous maps of the form $X \stackrel{p}{\leftarrow} \Gamma \stackrel{q}{\rightarrow} Y$ is called a selected pair of $\phi$ (written $(p, q) \subset \phi)$ if the following two conditions hold:

(i). $p$ is a Vietoris map

and

(ii). $q\left(p^{-1}(x)\right) \subset \phi(x)$ for any $x \in X$.

Now we define the admissible maps of Gorniewicz [9]. A upper semicontinuous map $\phi: X \rightarrow Y$ with compact values is said to be admissible (and we write $\phi \in A d(X, Y))$ provided there exists a selected pair $(p, q)$ of $\phi$. An example of an admissible map is a Kakutani map. A upper semicontinuous map $\phi: X \rightarrow K(Y)$ is said to Kakutani (and we write $\phi \in \operatorname{Kak}(X, Y)$ ); here $K(Y)$ denotes the family of nonempty, convex, compact subsets of $Y$.

The following class of maps will play a major role in this paper. Let $Z$ and $W$ be subsets of Hausdorff topological vector spaces $Y_{1}$ and $Y_{2}$ and $G$ a multifunction. We say $G \in \Phi^{\star}(Z, W)[2]$ if $W$ is convex and there exists a map $S: Z \rightarrow W$ with $S(x) \subseteq G(x)$ for $x \in Z, S(x) \neq \emptyset$ and has convex values for each $x \in Z$ and the fibre $S^{-1}(w)=\{z \in Z: w \in S(z)\}$ is open (in $Z$ ) for each $w \in W$.

Let $Q$ be a class of topological spaces. A space $Y$ is an extension space for $Q$ (written $Y \in E S(Q)$ ) if for any pair $(X, K)$ in $Q$ with $K \subseteq X$ closed, any continuous function $f_{0}: K \rightarrow Y$ extends to a continuous function $f: X \rightarrow Y$. A space $Y$ is an approximate extension space for $Q$ (written $Y \in A E S(Q)$ ) if for any $\alpha \in \operatorname{Cov}(Y)$ and any pair $(X, K)$ in $Q$ with $K \subseteq X$ closed, and any continuous function $f_{0}: K \rightarrow Y$ there exists a continuous function $f: X \rightarrow Y$ such that $\left.f\right|_{K}$ is $\alpha$-close to $f_{0}$.

Let $V$ be a subset of a Hausdorff topological vector space $E$. Then we say $V$ is Schauder admissible if for every compact subset $K$ of $V$ and every covering $\alpha \in \operatorname{Cov}_{V}(K)$ there exists a continuous functions $\pi_{\alpha}: K \rightarrow V$ such that

(i). $\pi_{\alpha}$ and $i: K \rightarrow V$ are $\alpha$-close;

(ii). $\pi_{\alpha}(K)$ is contained in a subset $C \subseteq V$ with $C \in A E S$ (compact).

Our first result is taken from $[1,10]$. 
Theorem 1.1. Let $X$ be a Schauder admissible subset of a Hausdorff topological vector space and $\Psi \in A D(X, X)$ a compact map. Then there exists a $x \in X$ with $x \in \Psi(x)$.

Remark 1.2. Other variations of Theorem 1.1 can be found in [11].

We recall that a point $x \in X$ is a maximal element of a set valued map $F$ from a topological space $X$ to another topological space $Y$ if $F(x)=\emptyset$.

\section{MAXIMAL ELEMENT RESULTS}

We begin by establishing a new collectively fixed point result (motivated in part from $[12,14])$.

Theorem 2.1. Let $\left\{X_{i}\right\}_{i=1}^{N}$ be a family of convex compact sets each in a Hausdorff topological vector space $E_{i}$. For each $i \in\{1, \ldots, N\}$ suppose $F_{i}: X \equiv \prod_{i=1}^{N} X_{i} \rightarrow X_{i}$ and in addition there exists a map $S_{i}: X \rightarrow X_{i}$ with $S_{i}(x) \subseteq F_{i}(x)$ for $x \in X, S_{i}(x)$ has convex values for $x \in X$ and $S_{i}^{-1}(w)$ is open (in $X$ ) for each $w \in X_{i}$. Finally suppose for each $x \in X$ there exists a $i \in\{1, \ldots, N\}$ with $S_{i}(x) \neq \emptyset$. Then there exists $a x \in X$ and $a i \in\{1, . ., N\}$ with $x_{i} \in F_{i}(x)$ (here $x_{i}$ is the projection of $x$ on $X_{i}$ ).

Proof. Note $A_{i}=\left\{x \in X: S_{i}(x) \neq \emptyset\right\}, i \in\{1, . ., N\}$ is an open covering of $X$ (recall the fibres of $S_{i}$ are open). Now since $X$ is compact (so in particular paracompact) then from [8, Lemma 5.1.6, pp301] there exists a covering $\left\{B_{i}\right\}_{i=1}^{N}$ of $X$ where $B_{i}$ is closed and $B_{i} \subset A_{i}$ for all $i \in\{1, \ldots, N\}$. For each $i \in\{1, . ., N\}$ let $G_{i}: X \rightarrow X_{i}$ and $T_{i}: X \rightarrow X_{i}$ be given by

$$
G_{i}(x)=\left\{\begin{array}{l}
F_{i}(x), x \in B_{i} \\
X_{i}, x \in X \backslash B_{i}
\end{array} \quad \text { and } T_{i}(x)=\left\{\begin{array}{l}
S_{i}(x), x \in B_{i} \\
X_{i}, x \in X \backslash B_{i} .
\end{array}\right.\right.
$$

We claim for $i \in\{1, \ldots, N\}$ that $G_{i} \in \Phi^{\star}\left(X, X_{i}\right)$. Note first for $i \in\{1, \ldots, N\}$ that $T_{i}(x) \neq \emptyset$ for $x \in X$. Also for $x \in X$ and $i \in\{1, \ldots, N\}$ then if $x \in B_{i}$ we have $T_{i}(x)=S_{i}(x) \subseteq F_{i}(x)=G_{i}(x)$ whereas if $x \in X \backslash B_{i}$ we have $T_{i}(x)=X_{i}=G_{i}(x)$. Also note if $y \in X_{i}$ then

$$
\begin{aligned}
T_{i}^{-1}(y) & =\left\{z \in X: y \in T_{i}(z)\right\} \\
& =\left\{z \in X \backslash B_{i}: y \in T_{i}(z)=X_{i}\right\} \cup\left\{z \in B_{i}: y \in T_{i}(z)\right\} \\
& =\left(X \backslash B_{i}\right) \cup\left\{z \in B_{i}: y \in S_{i}(z)\right\}=\left(X \backslash B_{i}\right) \cup\left[B_{i} \cap\left\{z \in X: y \in S_{i}(z)\right\}\right] \\
& =\left(X \backslash B_{i}\right) \cup\left[B_{i} \cap S_{i}^{-1}(y)\right]=X \cap\left[\left(X \backslash B_{i}\right) \cup S_{i}^{-1}(y)\right]=\left(X \backslash B_{i}\right) \cup S_{i}^{-1}(y)
\end{aligned}
$$

which is open in $X$ (note $S_{i}^{-1}(y)$ is open in $X$ and $B_{i}$ is closed in $X$ ). Thus for $i \in\{1, \ldots, N\}$ we have $G_{i} \in \Phi^{\star}\left(X, X_{i}\right)$. 
Now since $X$ is compact for each $i \in\{1, \ldots, N\}$ from $[2,5]$ there exists a continuous (single valued) selection $f_{i}: X \rightarrow X_{i}$ of $G_{i}$ with $f_{i}(x) \in T_{i}(x) \subseteq G_{i}(x)$ for $x \in X$ and also there exists a finite set $C_{i}$ of $X_{i}$ with $f_{i}(X) \subseteq c o\left(C_{i}\right) \equiv D_{i}$. Let

$$
D=\prod_{i=1}^{N} D_{i} \text { and } f(x)=\prod_{i=1}^{N} f_{i}(x), x \in D .
$$

Now $D$ is compact and convex, $f: D \rightarrow D$ and $f(D)$ lies in a finite dimensional subspace of $E=\prod_{i=1}^{N} E_{i}$. Brouwer's fixed point theorem guarantees that there exists a $x \in D$ with $x=f(x)$ i.e. $x_{j}=f_{j}(x) \in T_{j}(x) \subseteq G_{j}(x)$ for each $j \in\{1, . ., N\}$. Now since $\left\{B_{i}\right\}_{i=1}^{N}$ is a covering of $X$ there exists a $j_{0} \in\{1, . ., N\}$ with $x \in B_{j_{0}}$ so $x_{j_{0}} \in G_{j_{0}}(x)=F_{j_{0}}(x)$.

Remark 2.2. Note one could replace $\left\{X_{i}\right\}_{i=1}^{N}$ in Theorem 2.1 with $\left\{X_{i}\right\}_{i \in I}$ where $I$ is an index set. In Theorem 2.1 since $X \equiv \prod_{i \in I} X_{i}$ is compact (since we assume each $X_{i}$ is compact) then we could assume in the statement of Theorem 2.1 that there exists a finite subset $I_{0}$ of $I$ and for each $x \in X$ there exists a $i \in I_{0}$ with $S_{i}(x) \neq \emptyset$ so as a result one could rewrite the statement of Theorem 2.1. This remark could also be applied to the other results in this paper.

Next we will rewrite Theorem 2.1 as a maximal type element result.

Theorem 2.3. Let $\left\{X_{i}\right\}_{i=1}^{N}$ be a family of convex compact sets each in a Hausdorff topological vector space. For each $i \in\{1, \ldots, N\}$ suppose $F_{i}: X \equiv \prod_{i=1}^{N} X_{i} \rightarrow X_{i}$ and in addition there exists a map $S_{i}: X \rightarrow X_{i}$ with $S_{i}(x) \subseteq F_{i}(x)$ for $x \in X, S_{i}(x)$ has convex values for $x \in X$ and $S_{i}^{-1}(w)$ is open (in $X$ ) for each $w \in X_{i}$. Now suppose for all $i \in\{1, . ., N\}$ that $x_{i} \notin F_{i}(x)$ for each $x \in X$. Then there exists a $x \in X$ with $S_{i}(x)=\emptyset$ for all $i \in\{1, \ldots, N\}$.

Proof. Suppose the conclusion is false. Then for each $x \in X$ there exists a $i \in$ $\{1, \ldots, N\}$ with $S_{i}(x) \neq \emptyset$. Now Theorem 2.1 guarantees a $x \in X$ and a $i \in\{1, . ., N\}$ with $x_{i} \in F_{i}(x)$, a contradiction.

We now discuss a generalization of majorized mappings in the literature (see [3, $4,6,15])$. Let $Z$ and $W$ be sets in a Hausdorff topological vector space with $W$ convex and $Z$ compact. Suppose $H: Z \rightarrow W, J: Z \rightarrow W$ and for each $y \in Z$ assume there exists a map $A_{y}: Z \rightarrow W$ and an open set $U_{y}$ containing $y$ with $H(z) \subseteq A_{y}(z)$ for every $z \in U_{y}, A_{y}$ is convex valued, $\left(A_{y}\right)^{-1}(x)$ is open (in $Z$ ) for each $x \in W$ and $J(w) \cap A_{y}(w)=\emptyset$ for $w \in Z$. We now claim that there exists a map $T: Z \rightarrow W$ with $H(z) \subseteq T(z)$ for $z \in Z, T$ is convex valued, $T^{-1}(x)$ is open (in $Z$ ) for each $x \in W$ and $J(w) \cap T(w)=\emptyset$ for $w \in Z$. To see this note $\left\{U_{y}\right\}_{y \in Z}$ is an open covering of $Z$ and since $Z$ is compact there exists $[7,8]$ a finite set $\left\{y_{1}, \ldots, y_{n}\right\}$ (with $y_{i} \in Z$ for 
$i \in\{1, \ldots, n\})$ and an open covering $\left\{V_{y_{i}}\right\}_{i=1}^{n}$ of $Z$ with $y_{i} \in V_{y_{i}}$ and $\Omega_{y_{i}}=\overline{V_{y_{i}}} \subseteq U_{y_{i}}$ for $i \in\{1, \ldots, n\}$. Fix $i \in\{1, \ldots, n\}$ and let

$$
Q_{y_{i}}(z)=\left\{\begin{array}{l}
A_{y_{i}}(z), \quad z \in \Omega_{y_{i}} \\
W, \quad z \in Z \backslash \Omega_{y_{i}} .
\end{array}\right.
$$

Now $Q_{y_{i}}$ is convex valued and $H(z) \subseteq Q_{y_{i}}(z)$ for every $z \in Z$ (note if $z \in \Omega_{y_{i}}$ then since $\Omega_{y_{i}} \subseteq U_{y_{i}}$ and since $H(w) \subseteq A_{y_{i}}(w)$ for $w \in U_{y_{i}}$ we have $H(z) \subseteq Q_{y_{i}}(z)$ whereas if $z \in Z \backslash \Omega_{y_{i}}$ then it is immediate since $\left.Q_{y_{i}}(z)=W\right)$. Also note the argument in Theorem 2.1 guarantees for any $x \in W$ that

$$
\left(Q_{y_{i}}\right)^{-1}(x)=\left(Z \backslash \Omega_{y_{i}}\right) \cup\left(A_{y_{i}}\right)^{-1}(x)
$$

which is open in $Z$. Let $T: Z \rightarrow W$ be given by

$$
T(z)=\bigcap_{i=1}^{n} Q_{y_{i}}(z) \text { for } z \in Z .
$$

Now $T$ is convex valued, $H(z) \subseteq T(z)$ for every $z \in Z$ and for $x \in W$ we have

$$
\begin{aligned}
T^{-1}(x) & =\{z \in Z: x \in T(z)\}=\left\{z \in Z: x \in \bigcap_{i=1}^{n} Q_{y_{i}}(z)\right\} \\
& =\bigcap_{i=1}^{n}\left\{z \in Z: x \in Q_{y_{i}}(z)\right\}=\bigcap_{i=1}^{n}\left(Q_{y_{i}}\right)^{-1}(x)
\end{aligned}
$$

which is open in $Z$. Finally we note $J(w) \cap T(w)=\emptyset$ for $w \in Z$. To see this let $w \in Z$ and note there exists a $k \in\{1, \ldots, n\}$ with $y_{k} \in Z$ and $w \in \Omega_{y_{k}}$, so

$$
T(w)=\bigcap_{i=1}^{n} Q_{y_{i}}(w) \subseteq Q_{y_{k}}(w)=A_{y_{k}}(w)
$$

(since $\left.w \in \Omega_{y_{k}}\right)$ and thus $J(w) \cap T(w) \subseteq J(w) \cap A_{y_{k}}(w)=\emptyset$.

Now we will combine the above discussion with Theorem 2.3.

Theorem 2.4. Let $\left\{X_{i}\right\}_{i=1}^{N}$ be a family of convex compact sets each in a Hausdorff topological vector space. For each $i \in\{1, \ldots, N\}$ suppose $H_{i}: X \equiv \prod_{i=1}^{N} X_{i} \rightarrow X_{i}$ and for each $x \in X$ assume there exists a map $A_{i, x}: X \rightarrow X_{i}$ and an open set $U_{i, x}$ containing $x$ with $H_{i}(z) \subseteq A_{i, x}(z)$ for every $z \in U_{i, x}, A_{i, x}$ is convex valued, $\left(A_{i, x}\right)^{-1}(z)$ is open (in $X)$ for each $z \in X_{i}$ and $w_{i} \notin A_{i, x}(w)$ for each $w \in X$. Then there exists a $x \in X$ with $H_{i}(x)=\emptyset$ for all $i \in\{1, \ldots, N\}$.

Proof. Let $i \in\{1, \ldots, N\}$. From the discussion after Theorem 2.3 (with $Z=X, W=$ $X_{i}, H=H_{i}, J=$ Projection of $X$ on $\left.X_{i}, A_{y}=A_{i, x}\right)$ there exists a map $T_{i}: X \rightarrow X_{i}$ with $H_{i}(w) \subseteq T_{i}(w)$ for $w \in X, T_{i}$ is convex valued, $\left(T_{i}\right)^{-1}(z)$ is open for each $z \in X_{i}$ and $w_{i} \notin T_{i}(w)$ for each $w \in X$; here for a $i \in\{1, \ldots, N\}$ we have that $\left\{U_{i, x}\right\}_{x \in X}$ is an open covering of $X$ so there exists a finite set $\left\{y_{i, 1}, \ldots, y_{i, n_{i}}\right\}$ (with $y_{i, j} \in X$ for 
$\left.j \in\left\{1, \ldots, n_{i}\right\}\right)$ and an open covering $\left\{V_{i, y_{i, j}}\right\}_{i=1}^{n_{i}}$ of $X$ and $\Omega_{i, y_{i, j}}=\overline{V_{i, y_{i, j}}} \subseteq U_{i, y_{i, j}}$ for $j \in\left\{1, \ldots, n_{i}\right\}$ and for fixed $j \in\left\{1, \ldots, n_{i}\right\}$,

$$
Q_{i, y_{i, j}}(z)=\left\{\begin{array}{l}
A_{i, y_{i, j}}(z), \quad z \in \Omega_{i, y_{i, j}} \\
X_{i}, \quad z \in X \backslash \Omega_{i, y_{i, j}}
\end{array}\right.
$$

and

$$
T_{i}(z)=\bigcap_{j=1}^{n_{i}} Q_{i, y_{i, j}}(z) \text { for } z \in X
$$

Now we will apply Theorem 2.3 with $F_{i}=S_{i}=T_{i}$ and so there exists a $x \in X$ with $T_{j}(x)=\emptyset$ for all $j \in\{1, \ldots, N\}$. Now since $H_{j}(w) \subseteq T_{j}(w)$ for $w \in X$ then we have $H_{j}(x)=\emptyset$ for all $j \in\{1, \ldots, N\}$.

Next we will discuss collectively coincidence points motivated in part by $[4,13]$.

Theorem 2.5. Let $\left\{X_{i}\right\}_{i=1}^{N},\left\{Y_{i}\right\}_{i=1}^{N_{0}}$ be families of convex sets each in a Hausdorff topological vector space $E_{i}$ with $\prod_{i=1}^{N} X_{i}$ paracompact and in addition $\left\{Y_{i}\right\}_{i=1}^{N_{0}}$ is also a family of compact sets. For each $i \in\left\{1, \ldots, N_{0}\right\}$ suppose $F_{i}: X \equiv \prod_{i=1}^{N} X_{i} \rightarrow Y_{i}$ and there exists a map $T_{i}: X \rightarrow Y_{i}$ with $T_{i}(x) \subseteq F_{i}(x)$ for $x \in X, T_{i}(x)$ has convex values for each $x \in X$ and $T_{i}^{-1}(w)$ is open (in $X$ ) for each $w \in Y_{i}$. For each $j \in\{1, \ldots . N\}$ suppose $G_{j}: Y \equiv \prod_{i=1}^{N_{0}} Y_{i} \rightarrow X_{j}$ and there exists a map $S_{j}: Y \rightarrow X_{j}$ with $S_{j}(y) \subseteq G_{j}(y)$ for $y \in Y, S_{j}(y)$ has convex values for each $y \in Y$ and $S_{j}^{-1}(w)$ is open (in $Y$ ) for each $w \in X_{j}$. Finally suppose for each $x \in X$ there exists a $i \in\left\{1, \ldots, N_{0}\right\}$ with $T_{i}(x) \neq \emptyset$ and suppose for each $y \in Y$ there exists a $j \in\{1, \ldots, N\}$ with $S_{j}(y) \neq \emptyset$. Then there exists $a x \in X, a y \in Y, a j_{0} \in\left\{1, \ldots, N_{0}\right\}$ and $a$ $i_{0} \in\{1, \ldots, N\}$ with $y_{j_{0}} \in F_{j_{0}}(x)$ and $x_{i_{0}} \in G_{i_{0}}(y)$.

Proof. Note $A_{i}=\left\{x \in X: T_{i}(x) \neq \emptyset\right\}, i \in\left\{1, . ., N_{0}\right\}$ is an open covering of $X$ so from [8, Lemma 5.1.6, pp301] there exists a covering $\left\{B_{i}\right\}_{i=1}^{N_{0}}$ of $X$ where $B_{i}$ is closed in $X$ and $B_{i} \subset A_{i}$ for all $i \in\left\{1, \ldots, N_{0}\right\}$. Also $C_{i}=\left\{y \in Y: S_{i}(y) \neq \emptyset\right\}, i \in\{1, . ., N\}$ is an open covering of $Y$ and from [8, Lemma 5.1.6, pp301] there exists a covering $\left\{D_{i}\right\}_{i=1}^{N}$ of $Y$ where $D_{i}$ is closed in $Y$ and $D_{i} \subset C_{i}$ for all $i \in\{1, \ldots, N\}$. Now for each $i \in\left\{1, . ., N_{0}\right\}$ let $H_{i}: X \rightarrow Y_{i}$ and $J_{i}: X \rightarrow Y_{i}$ be given by

$$
H_{i}(x)=\left\{\begin{array}{l}
F_{i}(x), x \in B_{i} \\
Y_{i}, x \in X \backslash B_{i}
\end{array} \text { and } J_{i}(x)=\left\{\begin{array}{l}
T_{i}(x), x \in B_{i} \\
Y_{i}, x \in X \backslash B_{i} .
\end{array}\right.\right.
$$

Also for each $i \in\{1, . ., N\}$ let $M_{i}: Y \rightarrow X_{i}$ and $L_{i}: Y \rightarrow X_{i}$ be given by

$$
M_{i}(y)=\left\{\begin{array}{l}
G_{i}(y), y \in D_{i} \\
X_{i}, y \in Y \backslash D_{i}
\end{array} \quad \text { and } \quad L_{i}(y)=\left\{\begin{array}{l}
S_{i}(y), y \in D_{i} \\
X_{i}, y \in Y \backslash D_{i}
\end{array}\right.\right.
$$

The reasoning in Theorem 2.1 guarantees that $H_{i} \in \Phi^{\star}\left(X, Y_{i}\right)$ for $i \in\left\{1, \ldots, N_{0}\right\}$ and $M_{i} \in \Phi^{\star}\left(Y, X_{i}\right)$ for $i \in\{1, \ldots, N\}$. 
Now since $Y$ is compact for each $i \in\{1, \ldots, N\}$ from $[2,5]$ there exists a continuous (single valued) selection $q_{i}: Y \rightarrow X_{i}$ of $M_{i}$ with $q_{i}(y) \in L_{i}(y) \subseteq M_{i}(y)$ for $y \in Y$ and there exists a finite subset $R_{i}$ of $X_{i}$ with $q_{i}(Y) \subseteq \operatorname{co}\left(R_{i}\right) \equiv Q_{i}$. Let $Q=\prod_{i=1}^{N} Q_{i}(\subseteq$ $X)$ and note $Q$ is compact. Let $H_{i}^{\star}$ (respectively, $J_{i}^{\star}$ ) denote the restriction of $H_{i}$ (respectively, $\left.J_{i}\right)$ to $Q$. Note for $i \in\left\{1, \ldots, N_{0}\right\}$ that $H_{i}^{\star} \in \Phi^{\star}\left(Q, Y_{i}\right)$ since for $y \in Y_{i}$ we have

$$
\begin{aligned}
\left(J_{i}^{\star}\right)^{-1}(y) & =\left\{z \in Q: y \in J_{i}^{\star}(z)\right\}=\left\{z \in Q: y \in J_{i}(z)\right\} \\
& =Q \cap\left\{z \in X: y \in J_{i}(z)\right\}=Q \cap J_{i}^{-1}(y)
\end{aligned}
$$

which is open in $Q \cap X=Q$. Now since $Q$ is compact (in particular paracompact) for each $i \in\left\{1, \ldots, N_{0}\right\}$ from $[2,5]$ there exists a continuous (single valued) selection $h_{i}: Q \rightarrow Y_{i}$ of $H_{i}^{\star}$ with $h_{i}(x) \in J_{i}^{\star}(x) \subseteq H_{i}^{\star}(x)$ for $x \in Q$. Let

$$
h(x)=\prod_{i=1}^{N_{0}} h_{i}(x) \text { for } x \in Q \text { and } q(y)=\prod_{i=1}^{N} q_{i}(y) \text { for } y \in Y
$$

and note $h: Q \rightarrow Y$ and $q: Y \rightarrow Q$ are continuous. Consider the continuous map $\theta: Q \rightarrow Q$ given by $\theta(x)=q(h(x))$ for $x \in Q$. Note $Q$ is a compact convex subset in a finite dimensional subspace of $E=\prod_{i=1}^{N} E_{i}$ so Brouwer's fixed point theorem guarantees that there exists a $x \in Q$ with $x=\theta(x)=q(h(x))$. Let $y=h(x)$ so $x=q(y)$. Then since $x \in Q$ we have $y_{j}=h_{j}(x) \in J_{j}^{\star}(x) \subseteq H_{j}^{\star}(x)$ i.e. $y_{j} \in H_{j}(x)$ for $j \in\left\{1, \ldots, N_{0}\right\}$ and $x_{i}=q_{i}(y) \in L_{i}(y) \subseteq M_{i}(y)$ for $i \in\{1, \ldots, N\}$. Next since $\left\{B_{i}\right\}_{i=1}^{N_{0}}$ is a covering of $X$ there exists a $j_{0} \in\left\{1, . ., N_{0}\right\}$ with $x \in B_{j_{0}}$ so $y_{j_{0}} \in H_{j_{0}}(x)=F_{j_{0}}(x)$. Finally we note since $\left\{D_{i}\right\}_{i=1}^{N}$ is a covering of $Y$ there exists a $i_{0} \in\{1, . ., N\}$ with $x \in D_{i_{0}}$ so $x_{i_{0}} \in M_{i_{0}}(y)=G_{i_{0}}(y)$.

Theorem 2.6. Let $\left\{X_{i}\right\}_{i=1}^{N},\left\{Y_{i}\right\}_{i=1}^{N_{0}}$ be families of convex sets each in a Hausdorff topological vector space with $\prod_{i=1}^{N} X_{i}$ paracompact and in addition $\left\{Y_{i}\right\}_{i=1}^{N_{0}}$ is also a family of compact sets. For each $i \in\left\{1, \ldots, N_{0}\right\}$ suppose $F_{i}: X \equiv \prod_{i=1}^{N} X_{i} \rightarrow Y_{i}$ and there exists a map $T_{i}: X \rightarrow Y_{i}$ with $T_{i}(x) \subseteq F_{i}(x)$ for $x \in X, T_{i}(x)$ has convex values for each $x \in X$ and $T_{i}^{-1}(w)$ is open (in $\left.X\right)$ for each $w \in Y_{i}$. For each $j \in\{1, \ldots . . N\}$ suppose $G_{j}: Y \equiv \prod_{i=1}^{N_{0}} Y_{i} \rightarrow X_{j}$ and there exists a map $S_{j}: Y \rightarrow X_{j}$ with $S_{j}(y) \subseteq G_{j}(y)$ for $y \in Y, S_{j}(y)$ has convex values for each $y \in Y$ and $S_{j}^{-1}(w)$ is open (in $Y$ ) for each $w \in X_{j}$. Now suppose either for all $j \in\left\{1, \ldots, N_{0}\right\}$ we have $y_{j} \notin F_{j}(x)$ for each $(x, y) \in X \times Y$ or for all $i \in\{1, \ldots, N\}$ we have $x_{i} \notin G_{i}(y)$ for each $(x, y) \in X \times Y$. Then either there exists a $x \in X$ with $T_{i}(x)=\emptyset$ for all $i \in\left\{1, \ldots, N_{0}\right\}$ or there exists a $y \in Y$ with $S_{j}(y)=\emptyset$ for all $j \in\{1, \ldots, N\}$.

Proof. Suppose the conclusion is false. Then for each $x \in X$ there exists a $i \in$ $\left\{1, \ldots, N_{0}\right\}$ with $T_{i}(x) \neq \emptyset$ and for each $y \in Y$ there exists a $j \in\{1, \ldots, N\}$ with $S_{j}(y) \neq \emptyset$. Now Theorem 2.5 guarantees a $x \in X$, a $y \in Y$, a $j_{0} \in\left\{1, \ldots, N_{0}\right\}$ and a $i_{0} \in\{1, \ldots, N\}$ with $y_{j_{0}} \in F_{j_{0}}(x)$ and $x_{i_{0}} \in G_{i_{0}}(y)$, a contradiction. 
Theorem 2.7. Let $\left\{X_{i}\right\}_{i=1}^{N},\left\{Y_{i}\right\}_{i=1}^{N_{0}}$ be families of convex compact sets each in a Hausdorff topological vector space. For each $i \in\left\{1, \ldots, N_{0}\right\}$ and for each $j \in\{1, \ldots, N\}$ suppose $H_{i}: X \equiv \prod_{i=1}^{N} X_{i} \rightarrow Y_{i}$ and $\Psi_{j}: Y \equiv \prod_{i=1}^{N_{0}} Y_{i} \rightarrow X_{j}$ and for each $x \in X$ assume there exists a map $A_{i, x}: X \rightarrow Y_{i}$ and an open set $U_{i, x}$ containing $x$ with $H_{i}(z) \subseteq A_{i, x}(z)$ for every $z \in U_{i, x}, A_{i, x}$ is convex valued, $\left(A_{i, x}\right)^{-1}(z)$ is open (in $\left.X\right)$ for each $z \in Y_{i}$ and for each $y \in Y$ assume there exists a map $B_{j, y}: Y \rightarrow X_{j}$ and an open set $O_{j, y}$ containing y with $\Psi_{j}(z) \subseteq B_{j, y}(z)$ for every $z \in O_{j, y}, B_{j, y}$ is convex valued, $\left(B_{j, y}\right)^{-1}(z)$ is open (in $Y$ ) for each $z \in X_{j}$ and also assume either for all $i \in\left\{1, \ldots, N_{0}\right\}$ we have $v_{i} \notin A_{i, x}(u)$ for each $(u, v) \in X \times Y$ or for all $j \in\{1, \ldots, N\}$ we have $u_{j} \notin B_{j, y}(v)$ for each $(u, v) \in X \times Y$. Then either there exists a $x \in X$ with $H_{i}(x)=\emptyset$ for all $i \in\left\{1, \ldots, N_{0}\right\}$ or there exists a $y \in Y$ with $\Psi_{j}(y)=\emptyset$ for all $j \in\{1, \ldots, N\}$.

Proof. We will modify slightly the ideas in the discussion after Theorem 2.3. Fix $i \in\left\{1, . ., N_{0}\right\}$ (respectively, $j \in\{1, . ., N\}$ ). Note $\left\{U_{i, x}\right\}_{x \in X}$ is an open covering of $X$ (respectively, $\left\{O_{j, y}\right\}_{y \in Y}$ is an open covering of $Y$ ) so there exists a finite set $\left\{x_{i, 1}, \ldots, x_{i, n_{i}}\right\}$ (with $x_{i, j} \in X$ for $j \in\left\{1, \ldots, n_{i}\right\}$ ) and an open covering $\left\{V_{i, x_{i, k}}\right\}_{k=1}^{n_{i}}$ of $X$ with $x_{i, k} \in V_{i, x_{i, k}}$ and $\Omega_{i, x_{i, k}}=\overline{V_{i, x_{i, k}}} \subseteq U_{i, x_{i, k}}$ (respectively, a finite set $\left\{y_{j, 1}, \ldots, y_{j, n_{j}}\right\}$ and an open covering $\left\{C_{j, y_{j, l}}\right\}_{l=1}^{n_{j}}$ of $Y$ with $y_{j, l} \in C_{j, y_{j, l}}$ and $D_{j, y_{j, l}}=\overline{C_{j, y_{j, l}}} \subseteq O_{j, y_{j, l}}$ ) and for fixed $k \in\left\{1, \ldots, n_{i}\right\}$,

$$
Q_{i, x_{i, k}}(z)=\left\{\begin{array}{l}
A_{i, x_{i, k}}(z), \quad z \in \Omega_{i, x_{i, k}} \\
Y_{i}, \quad z \in X \backslash \Omega_{i, x_{i, k}}
\end{array}\right.
$$

and let $T_{i}: X \rightarrow Y_{i}$ be

$$
T_{i}(z)=\bigcap_{k=1}^{n_{i}} Q_{i, x_{i, k}}(z), z \in X
$$

(respectively, for fixed $l \in\left\{1, \ldots, n_{j}\right\}$,

$$
R_{j, y_{j, l}}(z)=\left\{\begin{array}{l}
B_{j, y_{j, l}}(z), \quad z \in D_{j, y_{j, l}} \\
X_{j}, \quad z \in Y \backslash D_{j, y_{j, l}}
\end{array}\right.
$$

and let $S_{j}: Y \rightarrow X_{j}$ be

$$
\left.S_{j}(w)=\bigcap_{l=1}^{n_{j}} R_{j, y_{j, l}}(w), w \in Y\right) .
$$

The argument in the discussion after Theorem 2.3 guarantees that $H_{i}(z) \subseteq T_{i}(z)$ for every $z \in X$ (respectively, $\Psi_{j}(w) \subseteq S_{j}(w)$ for $w \in Y$ ), $T_{i}$ (respectively, $S_{j}$ ) is convex valued and $T_{i}^{-1}(w)$ is open for each $w \in Y_{i}$ (respectively, $S_{j}^{-1}(z)$ is open for each $\left.z \in X_{j}\right)$.

There are two cases to consider (see the statement of Theorem 2.7). Suppose first that for each $x \in X$ for all $i \in\left\{1, \ldots, N_{0}\right\}$ we have $v_{i} \notin A_{i, x}(u)$ for each $(u, v) \in X \times Y$. Then for all $i \in\left\{1, \ldots, N_{0}\right\}$ we have $v_{i} \notin T_{i}(u)$ for each $(u, v) \in X \times Y$; to see this fix 
$i \in\left\{1, \ldots, N_{0}\right\}$ and $(u, v) \in X \times Y$ and note there exists a $x_{i, m}\left(m \in\left\{1, \ldots, n_{i}\right\}\right)$ with $u \in \Omega_{i, x_{i, m}}$ so

$$
T_{i}(u)=\bigcap_{k=1}^{n_{i}} Q_{i, x_{i, k}}(u) \subseteq Q_{i, x_{i, m}}(u)=A_{i, x_{i, m}}(u)
$$

and as a result $v_{i} \notin T_{i}(u)$ since $v_{i} \notin A_{i, x_{i, m}}(u)$ and $T_{i}(u) \subseteq A_{i, x_{i, m}}(u)$. Next consider the case that for each $y \in Y$ for all $j \in\{1, \ldots, N\}$ we have $u_{j} \notin B_{j, y}(v)$ for each $(u, v) \in X \times Y$. As in the first case (with $S_{j}$ replacing $T_{i}$ ) we obtain for all $j \in$ $\{1, \ldots, N\}$ we have $u_{j} \notin S_{j}(v)$ for each $(u, v) \in X \times Y$.

Now apply Theorem 2.6 (with $F_{i}=T_{i}$ and $G_{j}=S_{j}$ ) so either there exists a $x \in X$ with $T_{i}(x)=\emptyset$ for all $i \in\left\{1, \ldots, N_{0}\right\}$ or there exists a $y \in Y$ with $S_{j}(y)=\emptyset$ for all $j \in\{1, \ldots, N\}$, Now since $H_{i}(z) \subseteq T_{i}(z), z \in X$ and $\Psi_{j}(w) \subseteq S_{j}(w), w \in Y$ the conclusion follows.

Remark 2.8. In Theorem 2.7 we could replace $\left\{X_{i}\right\}_{i=1}^{N}$ is a family of compact sets with the assumption that $X \equiv \prod_{i=1}^{N} X_{i}$ in paracompact. The proof is as in Theorem 2.7 (see Theorem 2.6) once we describe the map $T_{i}\left(i \in\left\{1, \ldots, N_{0}\right\}\right.$ fixed) as follows: Note $\left\{U_{i, x}\right\}_{x \in X}$ is an open covering of $X$ so there exists a locally finite open covering $\left\{V_{i, x}\right\}_{x \in X}$ of $X$ with $x \in V_{i, x}$ and $\Omega_{i, x}=\overline{V_{i, x}} \subseteq U_{i, x}$ for each $x \in X$, and for each $x \in X$ let

$$
Q_{i, x}(z)=\left\{\begin{array}{l}
A_{i, x}(z), \quad z \in \Omega_{i, x} \\
X_{i}, \quad z \in X \backslash \Omega_{i, x}
\end{array}\right.
$$

(it is easy to see that $Q_{i, x}$ is convex valued and $H_{i}(z) \subseteq Q_{i, x}(z)$ for $z \in X$ ) and let $T_{i}: X \rightarrow Y_{i}$ be

$$
T_{i}(z)=\bigcap_{x \in X} Q_{i, x}(z) \text { for } z \in X
$$

Note $H_{i}(z) \subseteq T_{i}(z)$ for $z \in X$ and $T_{i}$ is convex valued. It remains to show $T_{i}^{-1}(y)$ is open for each $y \in Y_{i}$. Fix $y \in Y_{i}$ and let $u \in T_{i}^{-1}(y)$. Since $\left\{V_{i, x}\right\}_{x \in X}$ is locally finite there exists an open neighborhood $N_{u}$ of $u$ such that $\left\{x \in X: N_{u} \cap V_{i, x} \neq \emptyset\right\}=$ $\left\{x_{i, 1}, \ldots, x_{i, m_{i}}\right\}$ (a finite set). Now if $x \notin\left\{x_{i, 1}, \ldots, x_{i, m_{i}}\right\}$ then $\emptyset=V_{i, x} \cap N_{u}=\Omega_{i, x} \cap N_{u}$ so $Q_{i, x}(z)=Y_{i}$ for all $z \in N_{u}$, and as a result

$$
T_{i}(z)=\bigcap_{x \in X} Q_{i, x}(z)=\bigcap_{j=1}^{m_{i}} Q_{i, x_{i, j}}(z) \text { for all } z \in N_{u} .
$$

Now $T_{i}^{-1}(y)=\left\{z \in X: y \in T_{i}(z)\right\}$ but note

$$
\left\{z \in N_{u}: y \in T_{i}(z)\right\}=\left\{z \in N_{u}: y \in \bigcap_{j=1}^{m_{i}} Q_{i, x_{i, j}}(z)\right\}=N_{u} \cap\left[\cap_{j=1}^{m_{i}}\left(Q_{i, x_{i, j}}\right)^{-1}(y)\right]
$$

SO

$$
u \in N_{u} \cap\left[\cap_{j=1}^{m_{i}}\left(Q_{i, x_{i, j}}\right)^{-1}(y)\right] \subseteq T_{i}^{-1}(y)
$$

so $T_{i}^{-1}(y)$ is open. To finish the proof in Theorem 2.7 there is as before two cases to consider (see the statement of Theorem 2.7). Suppose first that for each $x \in X$ for 
all $i \in\left\{1, \ldots, N_{0}\right\}$ we have $v_{i} \notin A_{i, x}(u)$ for each $(u, v) \in X \times Y$. Fix $i \in\left\{1, \ldots, N_{0}\right\}$ and $(u, v) \in X \times Y$ and note there exists a $x^{\star} \in X$ with $u \in \Omega_{i, x^{\star}}$ so

$$
T_{i}(z)=\bigcap_{x \in X} Q_{i, x}(z) \subseteq Q_{i, x^{\star}}(u)=A_{i, x^{\star}}(u)
$$

so $v_{i} \notin T_{i}(u)$. Thus for all $i \in\left\{1, \ldots, N_{0}\right\}$ we have $v_{i} \notin T_{i}(u)$ for each $(u, v) \in X \times Y$. Next consider the case that for each $y \in Y$ for all $j \in\{1, \ldots, N\}$ we have $u_{j} \notin B_{j, y}(v)$ for each $(u, v) \in X \times Y$. Then as in the proof of Theorem 2.7 we have for all $j \in\{1, \ldots, N\}$ that $u_{j} \notin S_{j}(v)$ for each $(u, v) \in X \times Y$. Now apply Theorem 2.6.

Now we consider a collectively coincidence result between the $\Phi^{\star}$ and $A d$ classes.

Theorem 2.9. Let $\left\{X_{i}\right\}_{i=1}^{N},\left\{Y_{i}\right\}_{i=1}^{N_{0}}$ be families of convex sets each in a Hausdorff topological vector space $E_{i}$ and in addition $\left\{Y_{i}\right\}_{i=1}^{N_{0}}$ is also a family of compact sets. For each $i \in\left\{1, \ldots, N_{0}\right\}$ suppose $F_{i}: X \equiv \prod_{i=1}^{N} X_{i} \rightarrow Y_{i}$ and $F_{i} \in A d\left(X, Y_{i}\right)$. For each $j \in\{1, \ldots . N\}$ suppose $G_{j}: Y \equiv \prod_{i=1}^{N_{0}} Y_{i} \rightarrow X_{j}$ and there exists a map $S_{j}: Y \rightarrow X_{j}$ with $S_{j}(y) \subseteq G_{j}(y)$ for $y \in Y, S_{j}(y)$ has convex values for each $y \in Y$ and $S_{j}^{-1}(w)$ is open (in $Y$ ) for each $w \in X_{j}$. Finally suppose for each $y \in Y$ there exists a $j \in\{1, \ldots, N\}$ with $S_{j}(y) \neq \emptyset$. Then there exists a $x \in X$, a $y \in Y$, a $i_{0} \in\{1, \ldots, N\}$ with $y_{j} \in F_{j}(x)$ for all $j \in\left\{1, \ldots, N_{0}\right\}$ and $x_{i_{0}} \in G_{i_{0}}(y)$.

Proof. For each $i \in\{1, \ldots, N\}$ let $C_{i}, D_{i}, M_{i}$ and $L_{i}$ be as in Theorem 2.5 and note $M_{i} \in \Phi^{\star}\left(Y, X_{i}\right)$. Now since $Y$ is compact for each $i \in\{1, \ldots, N\}$ from $[2,5]$ there exists a continuous (single valued) selection $q_{i}: Y \rightarrow X_{i}$ of $M_{i}$ with $q_{i}(y) \in L_{i}(y) \subseteq M_{i}(y)$ for $y \in Y$ and there exists a finite subset $R_{i}$ of $X_{i}$ with $q_{i}(Y) \subseteq \operatorname{co}\left(R_{i}\right) \equiv Q_{i}$. Let $Q=\prod_{i=1}^{N} Q_{i}(\subseteq X)$ and note $Q$ is compact. Let $F_{i}^{\star}$ denote the restriction of $F_{i}$ to $Q$ and let $F^{\star}(x)=\prod_{i=1}^{N_{0}} F_{i}^{\star}(x)$ for $x \in Q$. Since $A d$ is closed under compositions and also since a finite product of $A d$ maps is an $A d$ map [9] then $F^{\star} \in A d(Q, Y)$. Let $q(y)=\prod_{i=1}^{N} q_{i}(y)$ for $y \in Y$ and note $q: Y \rightarrow Q$ since $q_{i}: Y \rightarrow Q_{i}$ for $i \in\{1, \ldots, N\}$. Thus $q F^{\star} \in A d(Q, Q)$ and note $Q$ is a compact convex subset in a finite dimensional subspace of $E=\prod_{i=1}^{N} E_{i}$ so Theorem 1.1 guarantees a $x \in Q$ with $x \in q\left(F^{\star}(x)\right)$. Now let $y \in F^{\star}(x)$ with $x=q(y)$. Note $y \in F(x)$ so $y_{j} \in F_{j}(x)$ for all $j \in\left\{1, \ldots, N_{0}\right\}$. Also since $x \in Q$ we have $x_{i}=q_{i}(y) \in L_{i}(y) \subseteq M_{i}(y)$ for $i \in\{1, \ldots, N\}$. Now since $\left\{D_{i}\right\}_{i=1}^{N}$ is a covering of $Y$ then there exists a $i_{0} \in\left\{1, \ldots, N_{0}\right\}$ with $y \in D_{i_{0}}$ so $x_{i_{0}} \in M_{i_{0}}(y)=G_{i_{0}}(y)$.

Theorem 2.10. Let $\left\{X_{i}\right\}_{i=1}^{N},\left\{Y_{i}\right\}_{i=1}^{N_{0}}$ be families of convex sets each in a Hausdorff topological vector space and in addition $\left\{Y_{i}\right\}_{i=1}^{N_{0}}$ is also a family of compact sets. For each $i \in\left\{1, \ldots, N_{0}\right\}$ suppose $F_{i}: X \equiv \prod_{i=1}^{N} X_{i} \rightarrow Y_{i}$ and $F_{i} \in \operatorname{Ad}\left(X, Y_{i}\right)$. For each $j \in\{1, \ldots . . N\}$ suppose $G_{j}: Y \equiv \prod_{i=1}^{N_{0}} Y_{i} \rightarrow X_{j}$ and there exists a map $S_{j}: Y \rightarrow X_{j}$ with $S_{j}(y) \subseteq G_{j}(y)$ for $y \in Y, S_{j}(y)$ has convex values for each $y \in Y$ and $S_{j}^{-1}(w)$ is open (in $Y$ ) for each $w \in X_{j}$. Now suppose either for all $i \in\{1, \ldots, N\}$ we have 
$x_{i} \notin G_{i}(y)$ for each $(x, y) \in X \times Y$ or for each $(x, y) \in X \times Y$ there exists a $j \in\left\{1, \ldots, N_{0}\right\}$ with $y_{j} \notin F_{j}(x)$. Then there exists a $y \in Y$ with $S_{i}(y)=\emptyset$ for all $i \in\{1, \ldots, N\}$.

Proof. Suupose the conclusion is false. Then for each $y \in Y$ there exists a $j \in$ $\{1, \ldots, N\}$ with $S_{j}(y) \neq \emptyset$. Now Theorem 2.9 guarantees a $x \in X$, a $y \in Y$, a $i_{0} \in$ $\{1, \ldots, N\}$ with $y_{j} \in F_{j}(x)$ for all $j \in\left\{1, \ldots, N_{0}\right\}$ and $x_{i_{0}} \in G_{i_{0}}(y)$, a contradiction.

Theorem 2.11. Let $\left\{X_{i}\right\}_{i=1}^{N},\left\{Y_{i}\right\}_{i=1}^{N_{0}}$ be families of convex sets each in a Hausdorff topological vector space and in addition $\left\{Y_{i}\right\}_{i=1}^{N_{0}}$ is also a family of compact sets. For each $i \in\left\{1, \ldots, N_{0}\right\}$ suppose $F_{i}: X \equiv \prod_{i=1}^{N} X_{i} \rightarrow Y_{i}$ and $F_{i} \in A d\left(X, Y_{i}\right)$. For each $j \in\{1, \ldots . N\}$ suppose $\Psi_{j}: Y \equiv \prod_{i=1}^{N_{0}} Y_{i} \rightarrow X_{j}$ and for each $y \in Y$ assume there exists a map $B_{j, y}: Y \rightarrow X_{j}$ and an open set $O_{j, y}$ containing $y$ with $\Psi_{j}(z) \subseteq B_{j, y}(z)$ for every $z \in O_{j, y}, B_{j, y}$ is convex valued and $\left(B_{j, y}\right)^{-1}(z)$ is open (in $Y$ ) for each $z \in X_{j}$. Also suppose either for each $y \in Y$ for all $j \in\{1, \ldots, N\}$ we have $u_{j} \notin B_{j, y}(v)$ for each $(u, v) \in X \times Y$ or for each $(x, y) \in X \times Y$ there exists a $i \in\left\{1, \ldots, N_{0}\right\}$ with $y_{i} \notin F_{i}(x)$. Then there exists a $y \in Y$ with $\Psi_{j}(y)=\emptyset$ for all $j \in\{1, \ldots, N\}$.

Proof. Let $j \in\{1, \ldots, N\}$ and create $\left\{y_{j, 1}, \ldots, y_{j, n_{j}}\right\}, C_{j, y_{j, l}}, D_{j, y_{j, l}}, R_{j, y_{j, l}}\left(l \in\left\{1, \ldots, n_{j}\right\}\right)$ and $S_{j}$ as in Theorem 2.7. We now claim that for all $j \in\{1, \ldots, N\}$ we have $u_{j} \notin S_{j}(v)$ for each $(u, v) \in X \times Y$ if in the statement of Theorem 2.11 we have for each $y \in Y$ for all $j \in\{1, \ldots, N\}$ we have $u_{j} \notin B_{j, y}(v)$ for each $(u, v) \in X \times Y$. Note for a fixed $j \in\{1, \ldots, N\}$ and $(u, v) \times X \times Y$ note there exists a $y_{j, m}\left(m \in\left\{1, \ldots, n_{j}\right\}\right)$ with $v \in D_{j, y_{j, m}}$ so

$$
S_{j}(v)=\bigcap_{l=1}^{n_{j}} R_{j, y_{j, l}}(v) \subseteq R_{j, y_{j, m}}(v)=B_{j, y_{j, m}}(v)
$$

so $u_{j} \notin S_{j}(v)$. Thus our claim is true. Now apply Theorem 2.10 (with $G_{j}=S_{j}$ ) so there exists a $y \in Y$ with $S_{i}(y)=\emptyset$ for all $i \in\{1, \ldots, N\}$. The result follows since $\Psi_{j}(w) \subseteq S_{j}(w), w \in Y$

\section{REFERENCES}

[1] R.P. Agarwal, D.O'Regan and S. Park, Fixed point theory for multimaps in extension type spaces, J. Korean Math. Soc., 39(2002), 579-591.

[2] H. Ben-El-Mechaiekh, P. Deguire and A. Granas, Points fixes et coincidences pour les applications multivoques II (Applications de type $\Phi$ and $\Phi^{\star}$ ), C.R. Acad. Sc., 295(1982), 381-384.

[3] A. Borglin and H. Keiding, Existence of equilibrium actions and of equilibrium: a note on "new" existence theorems, J. Math. Econom., 3(1976), 313-316.

[4] P. Deguire, K.K. Tan and X.Z. Yaun, The study of maximal elements, fixed points for $L_{S^{-}}$ majorized mappings and their applications to minimax and variational inequalities in product topological spaces, Nonlinear Analysis, 37(1999), 933-951.

[5] X.P. Ding, W.K. Kim and K.K. Tan, A selection theorem and its applications, Bulletin Australian Math. Soc., 46(1992), 205-212. 
[6] X.P. Ding and K.K. Tan, On equilibria of non-compact generalized games, Jour. Math. Anal. Appl., 177(1993), 226-238.

[7] J. Dugundji, Topology, Allyn and Bacon, Boston, 1966.

[8] R. Engelking, General Topology, Heldermann Verlag, Berlin, 1989.

[9] L. Gorniewicz, Topological fixed point theory of multivalued mappings, Kluwer Acad. Publishers, Dordrecht, 1991.

[10] D. O'Regan, Fixed point theory for extension type spaces and essential maps on topological spaces, Fixed Point Theory and Applications, 1(2004), 13-20.

[11] D.O'Regan, Deterministic and random fixed points for maps on extension type spaces, Applicable Analysis, 97(2018), 1960-1966.

[12] D.O'Regan, Collectively fixed point theory in the compact and coercive cases, Analele Stiintifice ale Universitatii Ovidius Constanta, Seria Mathematica, accepted.

[13] D. O'Regan, Collectively coincidence type results and applications, Applicable Analysis, accepted.

[14] D.O'Regan, A note on collectively fixed and coincidence points, submitted.

[15] N.C. Yanelis and N.D. Prabhakar, Existence of maximal elements and equlibria in linear topological spaces, J. Math. Econom., 12(1983), 233-245. 